\title{
Influence of processing temperature on microstructure and microhardness of copper subjected to high-pressure torsion
}

\author{
XIE ZiLing ${ }^{1,2 *}$ XIE JiJia ${ }^{2}$, HONG YouShi ${ }^{2 *} \&$ WU XiaoLei ${ }^{2}$ \\ ${ }^{1}$ College of Architecture and Civil Engineering, University of Wenzhou, Wenzhou 325035, China; \\ ${ }^{2}$ State Key Laboratory of Nonlinear Mechanics, Institute of Mechanics, Chinese Academy of Sciences, Beijing 100190, China
}

Received November 30, 2009; accepted February 3, 2010

\begin{abstract}
$\mathrm{Cu}$ samples were subjected to high-pressure torsion (HPT) with up to 6 turns at room temperature (RT) and liquid nitrogen temperature (LNT), respectively. The effects of temperature on grain refinement and microhardness variation were investigated. For the samples after HPT processing at RT, the grain size reduced from $43 \mu \mathrm{m}$ to $265 \mathrm{~nm}$, and the Vickers microhardness increased from HV52 to HV140. However, for the samples after HPT processing at LNT, the value of microhardness reached its maximum of HV150 near the center of the sample and it decreased to HV80 at the periphery region. Microstructure observations revealed that HPT straining at LNT induced lamellar structures with thickness less than $100 \mathrm{~nm}$ appearing near the central region of the sample, but further deformation induced an inhomogeneous distribution of grain sizes, with submicrometer-sized grains embedded inside micrometer-sized grains. The submicrometer-sized grains with high dislocation density indicated their nonequilibrium nature. On the contrary, the micrometer-sized grains were nearly free of dislocation, without obvious deformation trace remaining in them. These images demonstrated that the appearance of micrometer-sized grains is the result of abnormal grain growth of the deformed fine grains.
\end{abstract}

copper, high pressure torsion (HPT), microstructure, grain size, microhardness, cryogenic temperature

Citation: Xie Z L, Xie J J, Hong Y S, et al. Influence of processing temperature on microstructure and microhardness of copper subjected to high-pressure torsion. Sci China Tech Sci, 2010, 53: 1534-1539, doi: 10.1007/s11431-010-3157-7

\section{Introduction}

The method of severe plastic deformation (SPD) is of great interest to the production of ultrafine-grain or nano-grain microstructures for metals and alloys [1-13]. Several SPD methods for producing bulk metals with sub-micrometer or nanometer grain size have been reported, e.g., high pressure torsion (HPT) [4, 5], equal channel angular pressing (ECAP) $[6,7]$ and accumulative roll bonding (ARB) [8]. Among these methods, HPT is especially attractive because it is capable of producing ultrafine grains, which are smaller than those achieved using other methods [9]. Early experi-

*Corresponding authors (email: xiezl@wzu.edu.cn; hongys@imech.ac.cn) mental evidence suggested that the extent of grain refinement depends on the strain applied [4,5], deformation temperature [4] and material parameters [10]. Till now, there are few researches directly on the role of processing temperature, especially at cryogenic temperatures, with respect to grain refinement during HPT. In this paper, a new type of HPT fixture equipped with an insulated vessel was designed and manufactured, which was then equipped with a large capacity testing machine. This system was capable of processing disc sample with a rather large thickness of $3.5 \mathrm{~mm}$ and a diameter of $12 \mathrm{~mm}$ at different temperatures. Cu samples were processed with this HPT apparatus up to 6 revolutions at room temperature (RT) and liquid nitrogen temperature (LNT), respectively. The deformed microstructure was observed by transmission electron microscopy (TEM) 
and optical microscopy (OM), and the microhardness was measured along the radius of samples. The influences of processing temperature on microstructure evolution and microhardness variation were investigated.

\section{Experimental}

$\mathrm{Cu}$ cylinders $(16 \mathrm{~mm}$ in diameter and $60 \mathrm{~mm}$ in height $)$ with a purity of $99.9 \mathrm{wt} \%$ were used as raw material for HPT treatment. Prior to the HPT treatment, the $\mathrm{Cu}$ cylinders were annealed in vacuum at $923 \mathrm{~K}$ for $3 \mathrm{~h}$ to diminish the effect of mechanical machining and obtain homogeneous coarse grains. The average grain size of the as-annealed sample was $43 \mu \mathrm{m}$ and annealing twins were observed in some grains, as shown in Figure 1. Disk samples were cut (from the cylinder) with diameter of $12 \mathrm{~mm}$ and thicknesses of $3.5 \mathrm{~mm}$, respectively. The sample was installed between the upper and the lower anvils of the HPT facility assembled with an insulated vessel, as illustrated in Figure 2. For the HPT processing at cryogenic temperatures, liquid-nitrogen (LN) was used as the cooling fluid. After a pressure of $1.2 \mathrm{GPa}$ on the sample, LN was injected into the insulated vessel until steady-state temperature obtained and

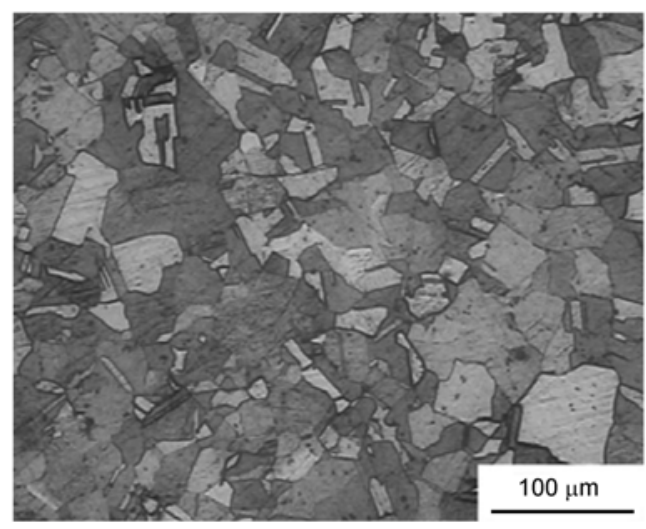

Figure 1 Optical micrograph of original $\mathrm{Cu}$ sample.

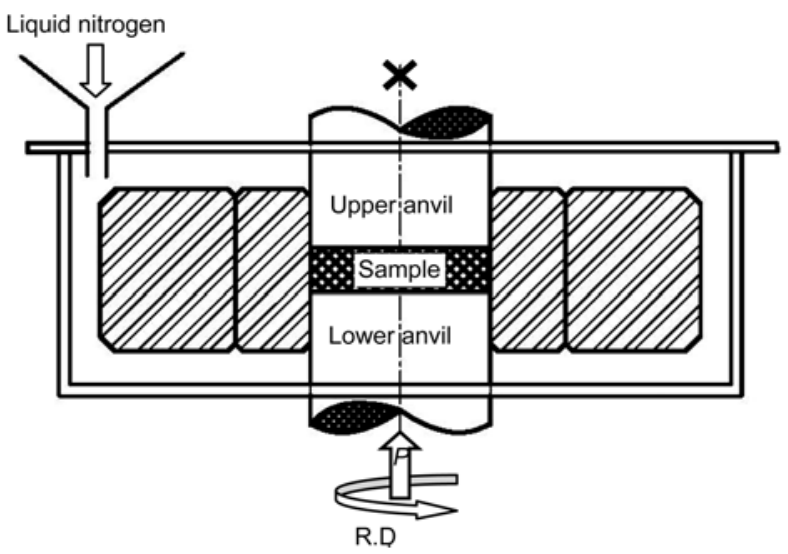

Figure 2 Schematic illustration of the device for HPT processing at RT and LNT. then the sample was strained with torsion at the speed of 0.25 revolutions per minute. During the HPT processing, $\mathrm{LN}$ was added to the insulated vessel for the full immersion of the HPT fixture to maintain the temperature of $-190^{\circ} \mathrm{C}$.

In the present experiments, disk samples were strained for 6 revolutions. Based on our observations, it was reasonable to assume that the thickness and the diameter of the sample kept the original sizes during HPT processing. Thus, the shear strain $\gamma$ was simply calculated by

$$
\gamma=2 \pi N r / h
$$

where $r$ is the distance from the center of the sample (rotation axis), $N$ is the number of revolutions and $h$ is the sample thickness. The dependence of $\gamma$ on $r$ is particularly helpful for this study because one may investigate material response in relation to different strain extents in a single sample.

An optical microscope (OM) was used to observe the microstructure of the deformed $\mathrm{Cu}$ samples. Observations were carried out in the longitudinal sections, which paralleled the torsion axis and contained the direction of the maximum shear, as shown in Figure 3 (section I). For OM observations, the samples were mechanically polished and etched in a solution containing $10 \mathrm{~g}$ ammonium persulfate and $100 \mathrm{~mL}$ distilled water for $10 \mathrm{~s}$. The detailed microstructures of the samples were characterized by transmission electron microscopy (TEM), performed on a JEOL100XL transmission electron microscope at an operating voltage of $100 \mathrm{kV}$. The thin foils for TEM observations were prepared by double-jet electrolytic polishing in an electrolyte consisting of $25 \%$ (volume ration) phosphoric acid, $25 \%$ ethanol and $50 \%$ distilled water at a voltage of 10-12 $\mathrm{V}$ and at RT. Measurement of the subgrain size and grain size were made directly from TEM micrographs using the linear intercept method.

Microhardness measurements were performed with an MH-5L Micro Hardness Tester. A load of $50 \mathrm{~g}$ was applied for $15 \mathrm{~s}$ in the longitudinal section, which contained the

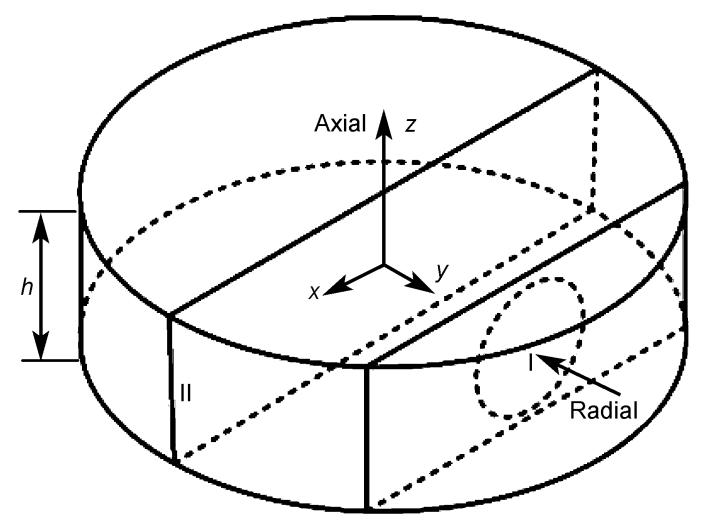

Figure 3 Schematic drawing of sampling positions ( $Z$ torsion axis), section I for microstructure observation (dashed circle representing TEM thin film), and section II for microhardness testing. 
torsion axis, as shown in Figure 3 (section II). At least 8 measurements were performed along the thickness of the sample for a given $x$ position.

\section{Results and discussion}

Micrographs from OM observations were shown in Figure 4. Development of the grain shape and grain refinement induced by HPT processing was clearly seen from these micrographs. At a relatively low strain level, as shown in Figures 4(a) and (c), the influence of deformation temperature on the pattern of deformation microstructure was not obvious. As compared to the original as-annealed samples, the equiaxed coarse grains were transformed into elongated grains under shear deformation. Further observations showed that parallel bands existed in some elongated grains in Figure 4(c). With increasing shear strain up to 32.3, a different deformation pattern was observed between the samples after HPT processed at RT and at LNT. HPT straining at RT induced obvious grain refinement with features difficult to be distinguished by $\mathrm{OM}$ and the elongated grains disappeared. However, further HPT straining at LNT made the elongated grains evolve into large grains with curved grain boundaries, without obvious grain refinement as compared to the original annealing state counterpart. Careful observation revealed that fine microstructures were present in the large grains (the dark-gray region in Figure 4(d)) which were formed after deformation by HPT at LNT.

Micrographs from TEM observations were shown in Figure 5. Development of the substructures and grain re- finement through subdivision induced by HPT processing was clearly seen from these micrographs. Detailed analysis for the mechanism of microstructure evolution in copper subjected to HPT processing at RT was proposed in refs. [4, 5]. The mechanism of microstructure evolution observed in the present sample deformed by HPT at RT was quite consistent with the previous knowledge, i.e., for those materials with medium or high stacking fault energy (SFE), such as $\mathrm{Fe}, \mathrm{Cu}$ and $\mathrm{Al}$, coarse grains are refined upon continued straining by various dislocation activities at low strain rates and at ambient temperatures. However, the present observation made in the longitudinal section of the HPT samples reveals a rather different deformation microstructure from those observed in the torsion plane as reported in previous study [5]. The observed deformation microstructure in the torsion plane was complex and it was generally characterized as having equiaxed morphology [5]. The present results showed that elongated subgrains with thickness of $200 \mathrm{~nm}$ formed at relative low strain level, and the elongated subgrains transformed into equiaxed grains with average grain size of $265 \mathrm{~nm}$ eventually, as shown in Figures 5(a), (b) and (c). It was easy to understand the different deformation patterns observed in torsion plane and longitudinal section by analyzing a unit geometric element during the torsion process. As demonstrated in Figure 6, the shape of initial unit configuration in torsion plane remained unchanged during the torsion process, while the initial unit configuration in the longitudinal section would be elongated under shear deformation. More details about the evolution procedure of elongated subgrains were demonstrated in ref. [13].
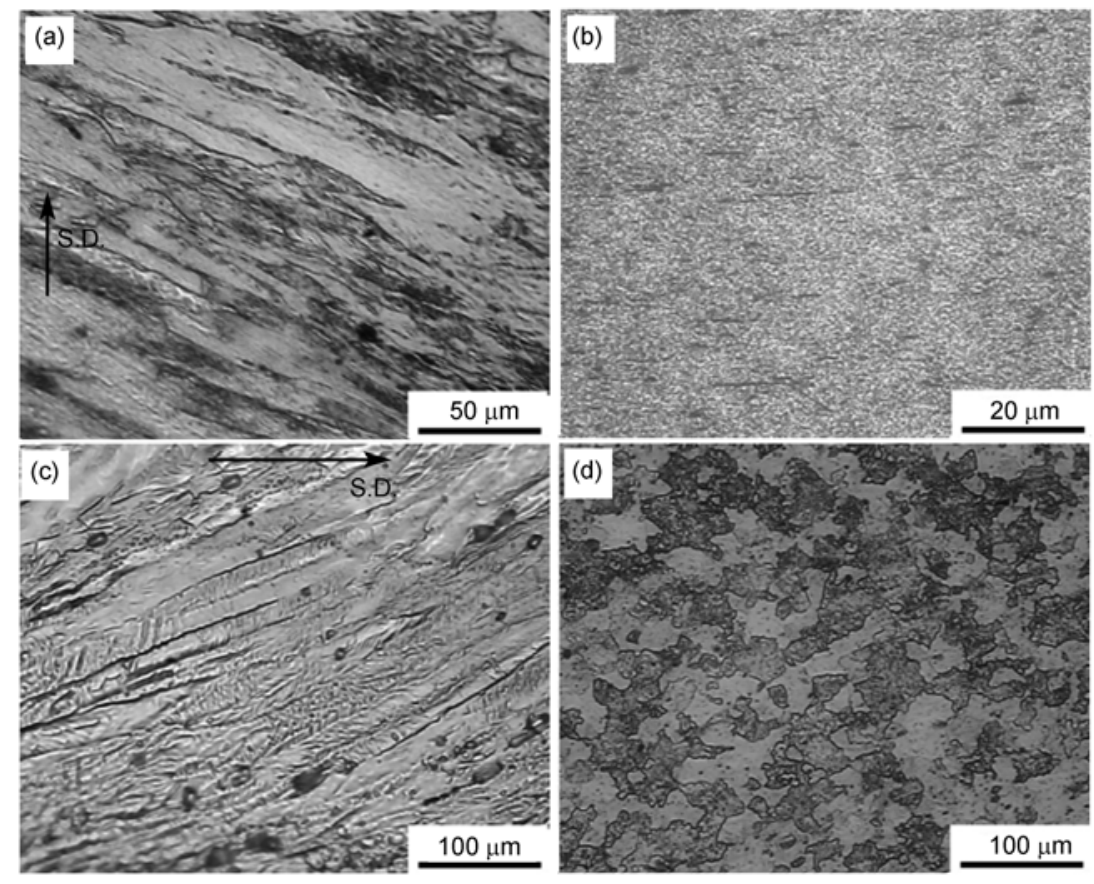

Figure 4 Optical micrographs of Cu samples after HPT processing up to 6 turns. (a) (b) HPT straining at RT; (c) (d) HPT straining at LNT $\gamma=5.4$ ( $r=0.5$ $\mathrm{mm}$ ) for (a) and (c); $\gamma=32.3(r=3 \mathrm{~mm})$ for (b) and (d). The black arrow indicates the shear direction, S.D). 

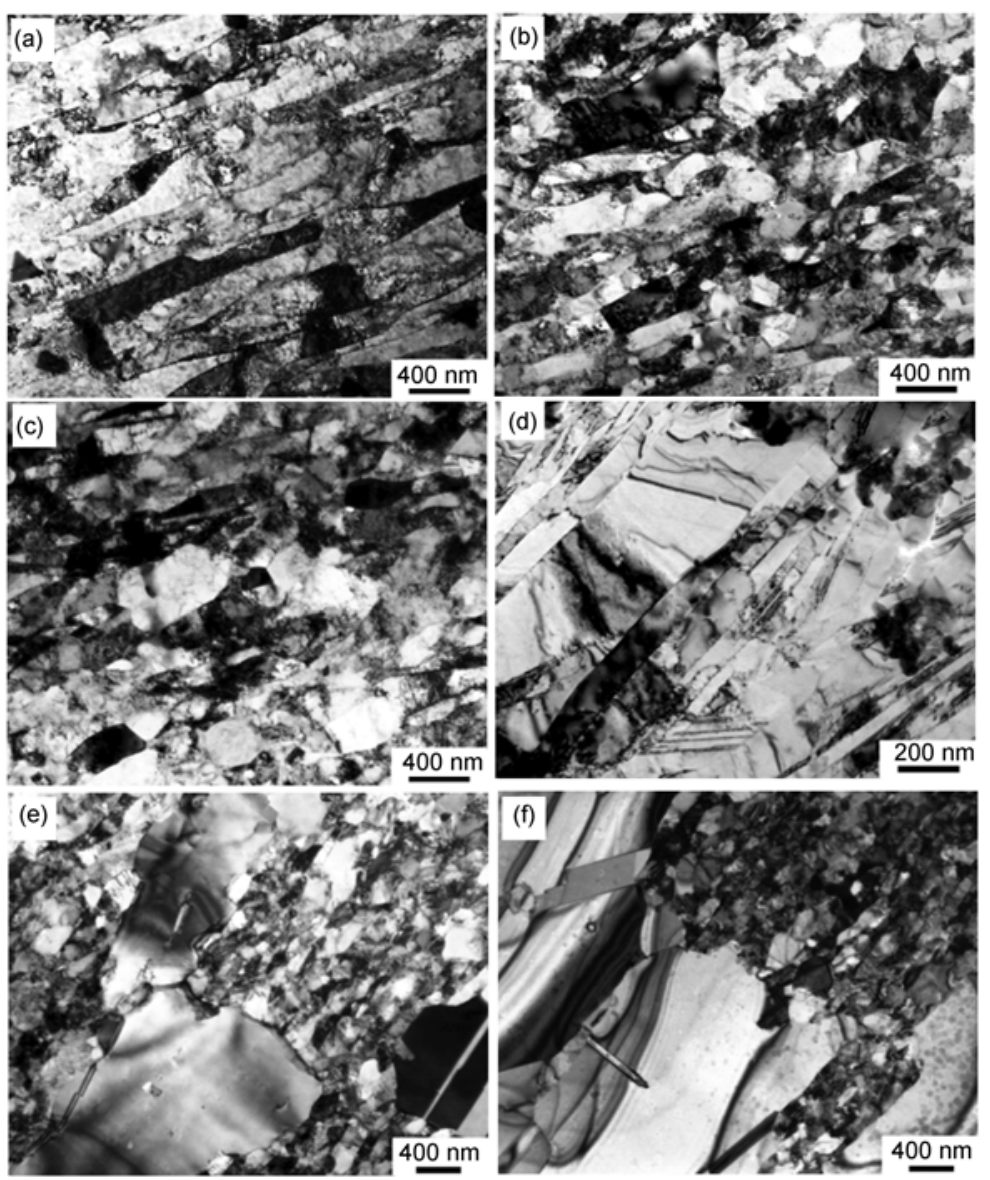

Figure 5 TEM micrographs of Cu samples after HPT processing up to 6 turns. (a) (b) (c) HPT straining at RT; (d) (e) (f) HPT straining at LNT ((a) and (d) with $\gamma=5.4(r=0.5 \mathrm{~mm})$, (b) and (e) with $\gamma=16.1(r=1.5 \mathrm{~mm})$, (c) and (f) with $\gamma=32.3(r=3 \mathrm{~mm})$ ).
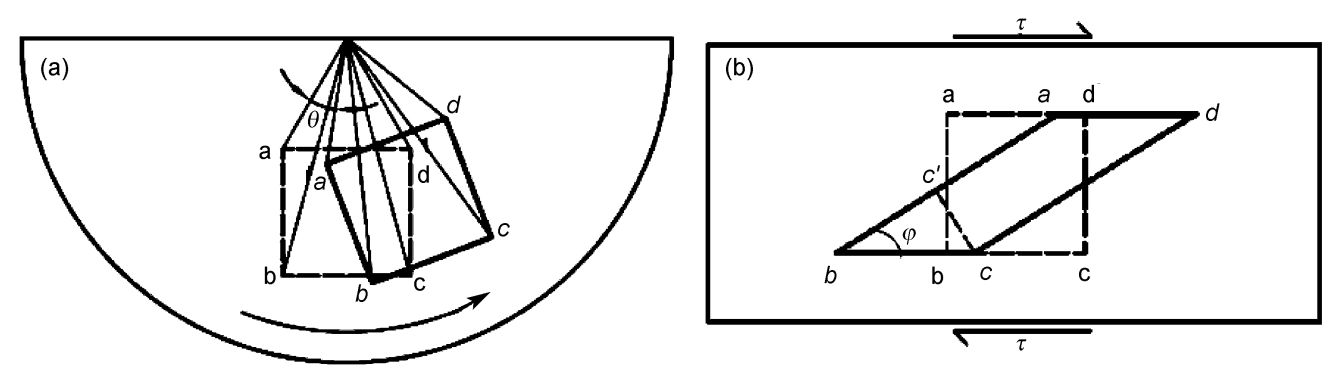

Figure 6 Schematic drawings of geometric changing in torsion plane (a) and longitudinal section (b) for samples deformed by HPT (abcd: initial unit configuration; abcd: deformed unit configuration).

HPT processed at LNT induced grain refinement more evidently at a low strain level, and lamellar subgains with thickness less than $100 \mathrm{~nm}$ formed near the torsion axis of the sample, as shown in Figure 5(d). With increasing strain, the inhomogeneous microstructure with submicrometersized grains embeded inside micrometer-sized grains was observed, as shown in Figure 5(e).The area fraction of the micrometer-sized grains reached $65 \%$. Further deformation induced more micrometer-sized grains with the disappearance of deformed small grains. Only occasionally, some deformed microstructures with the size smaller than $100 \mathrm{~nm}$ appeared around the micrometer-sized grains, as shown in Figure 5(f). It was clearly seen that the interior of micrometer-sized grains was quite clean, only some twins appeared, and no obvious deformation trace remained in them.

Figure 7 showed measured Vickers microhardness as a function of radius distance in section II of the $\mathrm{Cu}$ samples subjected to HPT up to 6 revolutions at RT and LNT. The measurements showed that along the radial direction the microhardness for the sample deformed by HPT at RT increased dramatically from the centre $(0-0.5 \mathrm{~mm})$ and then quickly reduced to a small incremental slope. 


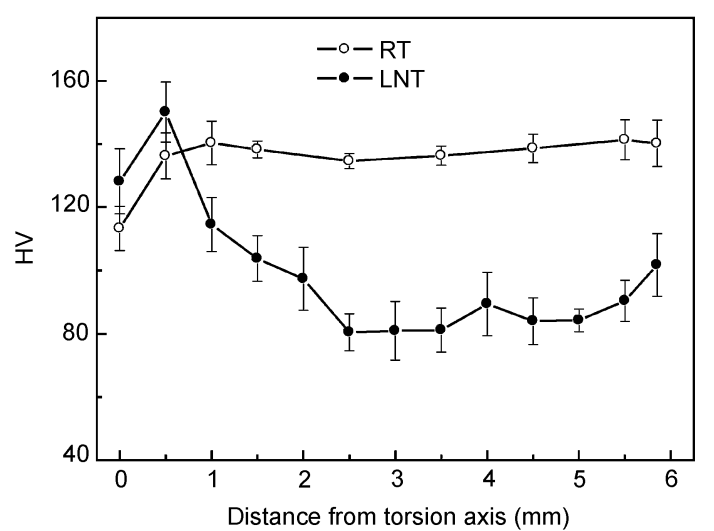

Figure 7 Measured Vickers microhardness as a function of radius distance in section II of copper samples subjected to HPT up to 6 revolutions at RT and LNT.

For the samples with HPT processing at LNT, the microhardness increased dramatically near the center of the sample and reached the maximum of HV 150 at $r=0.5 \mathrm{~mm}$, but decreased with increasing distance from torsion axis and reached a steady state of $\mathrm{HV} 80$ in the region of sample far from torsion axis. Referred to the microstructure observations, it was evident that the increase in hardness due to HPT processing was caused by grain refinement, and the decrease in hardness after further deformation at LNT was due to the existence of coarse grains.

Deformation-induced grain growth has been confirmed in nanocrystalline materials subjected to indentation, HPT processing and severe cyclic loading [14-17], but grain growth during HPT processing for coarse grain materials was rarely reported. Were the coarse grains observed in the sample after HPT straining at LNT the remainders of the initial grains? Indicated by eq. (1), an important feature in HPT is that the imposed strain varies across the sample and the strain near the center of sample is much smaller than that at the periphery regions. As a consequence, it is reasonable to anticipate that the grain refinement occurs at the periphery of sample at first. Based on the microstructure features along $r$ direction of the sample, as shown in Figures 4(c) and (d), grain refinement happened obviously near the center of the sample. It was then suggested that the coarse grains observed in the sample after HPT processing at LNT were not the remainders of original coarse grains.

It is known that the temperature at which recrystallization occurs decreases as the imposing strain increases. The stored energy, which provides the driving force for recrystallization, depends on such factors as composition (particularly impurity levels), grain size, and the extent and temperature of deformation [18]. Both nucleation and growth of grains are therefore more prone to occur at lower temperature in a deformed material with higher stored energy. This raises another question: Were the large grains observed in the $\mathrm{Cu}$ sample after deformed by HPT at LNT the results of secondary recrystallization of the fine grains due to elevated temperature? Before microstructure ob- servation, the samples experienced an increase in temperature by two processes. One was induced by HPT straining and the other was caused by sample exposure to environmental temperature post HPT processing at LNT. During HPT processing, the rotational speed was 0.25 revolutions per minute. Any heat generated in the sample was quickly conducted away into the HPT anvils, which were immersed in LN media. Thus the temperature increase in the sample during HPT was expected to be minimal, and not enough to elevate the temperature of sample to RT.

The constant-heating-rate DSC curve of the sample after HPT processed at LNT with a shear strain of 5.4 was displayed in Figure 8. The DSC result showed a single exothermic peak between $150^{\circ} \mathrm{C}$ and $200^{\circ} \mathrm{C}$, corresponding to the heat release due to recrystallization and subsequent grain growth. According to the results presented by ref. [19], a commercial purity $\mathrm{Cu}$ (higher than $99.9 \mathrm{wt} \%$ purity) was cold rolled at cryogenic temperatures $\left(-150^{\circ} \mathrm{C}--100^{\circ} \mathrm{C}\right)$ up to the strain of $30000 \%$, the recrystallization temperature of deformation microstructure was still higher than $150^{\circ} \mathrm{C}$. Hence the possibility of further HPT deformation at LNT to induce the $\mathrm{Cu}$ recrystallization temperature down to RT was very small, and the exposure of HPT deformed sample into environmental temperature would not induce abnormal grain growth. Thus the coarse grains observed in the samples after HPT processed at LNT for large strains were the results of abnormal grain growth during the deformation process, i.e. due to the process similar to dynamic recrystallization.

For metals with slow recovery process, such as those with low or medium SFE (copper, nickel and austenitic iron), dynamic recrystallization may take place when a critical deformation condition is reached. There are two classical dynamic recrystallization mechanisms, i.e., discontinuous dynamic recrystallization and continuous dynamic recrystallization [18]. The first mechanism is also known as migration dynamic recrystallization and characterized by the movement of preexisting high-angle grain boundaries through the deformed microstructures, leaving behind a strain free region in their wake. The second mechanism

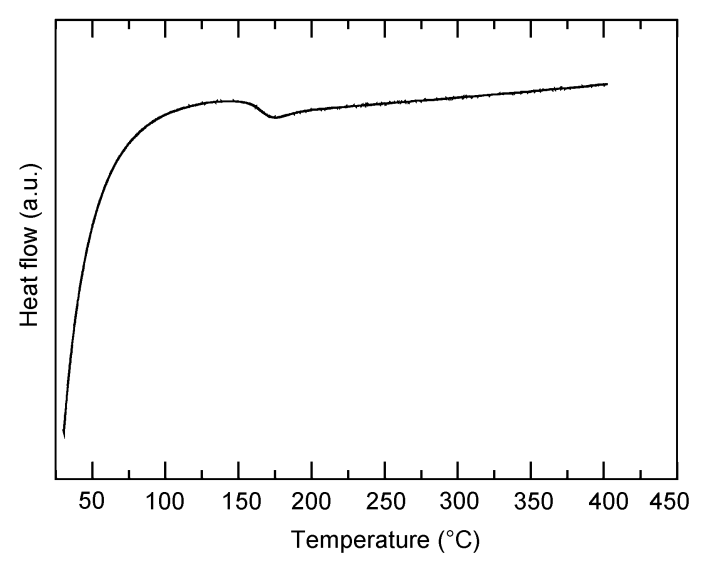

Figure 8 Differential scanning calorimetry (DSC) result at $20^{\circ} \mathrm{C} / \mathrm{min}$ of $\mathrm{Cu}$ sample after HPT processed at LNT with a shear stain of 5.4. 
includes geometric and progressive rotation recrystallization. The former forms new grains due to interpenetration of microbands and twins. The latter involves the gradual rotation of subgrains as the material is strained. Eventually, the misorientation between neighboring subgrains is sufficiently large that the sub-boundaries are turned into highangle grain boundaries [19].

The present results indicated that the evolution of microstructure in $\mathrm{Cu}$ sample deformed by HPT at RT was very similar to the geometric dynamic recrystallization process, resulting in the microstructure of small equiaxed grains of a size comparable with the subgrain size (Figures 5(a), (b), (c)). However, the coarse grains appearing in $\mathrm{Cu}$ sample deformed by HPT at LNT seemed to be the results of migration dynamic recrystallization. As shown in Figure 5(e), the bimodal grain size distributions appeared with micrometer-sized coarse grains embedded in the heavy deformed submicrometer-sized grains. The interior of the coarse grains was quite clean. During the HPT processing at LNT, the dynamic recovery process was reduced to allow the density of accumulated dislocations to reach a high level. A high strain hardening rate induces the flow stress to increase with further straining. When the stress reaches a critical value, stress-induced migration of grain boundaries occurs, resulting in a microstructure with dislocation-free grains coexistence with the grains with high dislocation density.

It was reported in ref. [16] that indentation at cryogenic temperatures might induce abnormal grain growth in $\mathrm{nc} \mathrm{Cu}$. Moreover, the grain growth was found faster at cryogenic temperature than at RT [16], and this suggested that the grain growth was driven primarily by stress, not by diffusion. It is known that during HPT processing at cryogenic temperature, the material is subjected to heavy plastic deformation with extremely high stress, which is the critical condition for deformation to induce grain growth in nanocrystalline materials [17].

Therefore, it is logical to assume that HPT processing at LNT induces grain refinement into nanometer at first, as shown in Figure 5(d), many twins in $10 \mathrm{~nm}$ appear in the elongated grains. Further straining induces an extremely high stress state in the nanostructures and stress-induced migration of grain boundaries occurs, resulting in bimodal grain size distributions.

\section{Conclusions}

$\mathrm{Cu}$ sample subjected to HPT at RT induces elongated grains with lamellar subgrains in them at relative low strain levels. With increasing strain, equiaxed grains with average size of $265 \mathrm{~nm}$ are the main features of the deformed microstructures. Vickers microhardness of the deformed sample increases from HV52 to HV140 due to grain refinement.

$\mathrm{Cu}$ sample subjected to HPT at LNT induces lamellar subgrains with twins near the center of the sample and the value of microhardness increases to HV150, but further straining induces bimodal grain size distributions, with submicrometer-sized grains embedded inside micrometer-sized grains. The submicrometer-sized grains comprise heavily deformed microstructure. However, the interior of micrometer-sized grains is quite clean, no obvious deformation trace remains in them. These images demonstrate that the appearance of micrometer-sized grains is the result of abnormal grain growth of the deformed fine grains.

This work was supported by the National Natural Science Foundation of China (Grant Nos. 10721202, 10772178, 50571110).

1 Valiev R Z, Islamgaliev R K, Alexandrov I V. Bulk nanostructured materials from severe plastic deformation. Prog Mater Sci, 2000, 45(2): 103-189

2 Lu K, Lu J. Surface nanocrystallization (SNC) of metallic materialspresentation of the concept behind a new approach. J Mater Sci Technol, 1999, 15(3): 193-197

3 Wu X L, Tao N R, Wei Q M, et al. Microstructural evolution and formation of nanocrystalline intermetallic compound during surface mechanical attrition treatment of cobalt. Acta Mater, 2007, 55(17): 5768-5779

4 Hebesberger T, Stüwe H P, Vorhauer A, et al. Structure of Cu deformed by high pressure torsion. Acta Mater, 2005, 53(2): 393-402

5 Zhilyaev A P, Langdon T G. Using high-pressure torsion for metal processing: Fundamentals and applications. Prog Mater Sci, 2008, 53(6): 893-979

6 Segal V M, Reznikov V I, Mulyukov N A. Plastic working of metals by simple shear. Russ Met, 1981, 1: 99-105

7 Wang J T, Kang S B, Kim H W, et al. Microstructural lamellae deformation and structural evolution in an $\mathrm{Al}-33 \% \mathrm{Cu}$ eutectic alloy during ECAP. J Mater Sci, 2002, 37(24): 5223-5227

8 Saito Y, Utsunomiya H, Tsuji N, et al. Novel ultra-high straining process for bulk materials - development of the accumulative roll-bonding (ARB) process. Acta Mater, 1999, 47(2): 579-583

9 Sakai G, Horita Z J, Langdon T G. Grain refinement and superplasticity in an aluminum alloy processed by high-pressure torsion. Mater Sci Eng A, 2005, 393(1-2): 344-351

10 Zhao Y H, Liao X Z, Zhu Y T, et al. Influence of stacking fault energy on nanostructure formation under high pressure torsion. Mater Sci Eng A, 2005, 410-411(25): 188-193

11 Zhang H W, Hansen N. Deformed metals and alloys with a structural scale from $5 \mathrm{~nm}$ to $100 \mathrm{~nm}$. J Mater Sci, 2007, 42(5): 1682-1693

12 Huang X, Winther G, Hansen N, et al. Microstructures of nickel deformed by high pressure torsion to high strains. Mater Sci Forum, 2003, 420-426: 2819-2824

13 Xie Z L, Xie J J, Wu X L, et al. Microstructures and compression properties of copper specimens deformed by high-pressure torsion (in Chinese). Acta Metall Sin, 2008, 44(7): 803-809

14 Schiøtz J. Strain-induced coarsening in nanocrystalline metals under cyclic deformation. Mater Sci Eng A, 2004, 375-377: 975-979

15 Zhang K, Weertman J R, Eastman J A. The influence of time, temperature, and grain size on indentation creep in high-purity nanocrystalline and ultrafine grain copper. Appl Phys Lett, 2004, 85: 5197-5199

16 Zhang K, Weertman J R, Eastman J A. Rapid stress-driven grain coarsening in nanocrystalline $\mathrm{Cu}$ at ambient and cryogenic temperatures. Appl Phys Lett, 2005, 87: 061921-3

17 Liao X Z, Kilmametov A R, Valiev R Z, et al. High-pressure torsion-induced grain growth in electrodeposited nanocrystalline Ni. Appl Phys Lett, 2006, 88: 021909-3

18 Humphreys F J, Hatherly M. Recrystallization and Related Annealing Phenomena. 2nd ed. New York: Pergamon Press, 2004. 11-15

19 Wang Y M, Jiao T, Ma E. Dynamic processes for nanostructure development in $\mathrm{Cu}$ after severe cryogenic rolling deformation. Mater Trans, 2003, 44(10): 1926-1934 